\title{
A new Wolf-Rayet galaxy with a Seyfert nucleus
}

\author{
Roberto Cid Fernandes ${ }^{1}$, R. Rodrigues Lacerda ${ }^{1}$, Henrique R. Schmitt ${ }^{2}$, \\ and Thaisa Storchi Bergmann ${ }^{3}$ \\ ${ }^{1}$ Departamento de Física, CFM - UFSC, Florianópolis, Brazil \\ ${ }^{2}$ Space Telescope Science Institute, Baltimore, USA \\ ${ }^{3}$ Instituto de Física, UFRGS, Porto Alegre, Brazil
}

\begin{abstract}
We report the detection of a WR feature in the nuclear spectrum of the Seyfert 2 galaxy Mrk 1210. The growing list of WR/Seyfert galaxies strengthens the evidence for a connection between nuclear activity and starformation.
\end{abstract}

\section{Mrk 1210, Wolf-Rayet stars and the starburst-AGN connection}

Mrk 1210 is a Seyfert 2 galaxy with a hidden Seyfert 1 nucleus, revealed through spectropolarimetry. As with such objects (e.g., Mrk 348, Mrk 477), the absence of strong broad lines and the polarization spectrum indicates that most of the optical light originates in an extended region, with a 'featureless' continuum resembling that of AGN (Tran 1995), but most likely due to circumnuclear starformation (Storchi Bergmann et al. 1998), possibly in the molecular torus itself (Cid Fernandes \& Terlevich 1995; Heckman et al. 1997). Disentangling the often similar signatures of star-formation and nuclear activity is a difficult task, but WR stars can be of great help. In Mrk 477, Heckman et al. (1997) detected the WR 'HeII'-bump, which, along with other (UV) features of massive stars conclusively proves the existence of a starburst in this Seyfert 2. Further detections of WRs in active galaxies have been recently reported (see T. Heckman, D. Kunth, these Proceedings; Tran et al. 1999).

Mrk 1210 is a further addition to this list. A broad WR bump is seen in its nuclear spectrum (Fig. 1), underlying narrow nebular HeII, [FeIII], [ArIv] and [NeIV] lines. The broad feature does not seem to be an artifact of blending of these lines. This was verified both with a gaussian decomposition and using the $\mathrm{H} \beta$, [OII] and [OIII] lines as profile templates for the deblending. In either case there remains a significant broad residual emission, but its equivalent width cannot be accurately measured $(E W<14 \AA)$. No other WR features are detected in the optical indicating that, as in Mrk 477, the WR's are predominantly WNL's.

The presence of a (hidden) AGN greatly complicates the comparison of the observed properties with models for starbursts and WR galaxies, as most of the nebular emission and even part of the continuum is powered by the AGN. We are currently analysing 2200-3300 $\AA$ HST and CTIO near-IR spectra of Mrk 1210, in search for other WR features to validate the optical detection and better define the WR subtype population. Observations of UV features normally used as diagnostic of the presence of massive stars (C IV $\lambda 1549$, SiIV+Orv] $\lambda 1397,1403, \mathrm{Nv}$ 


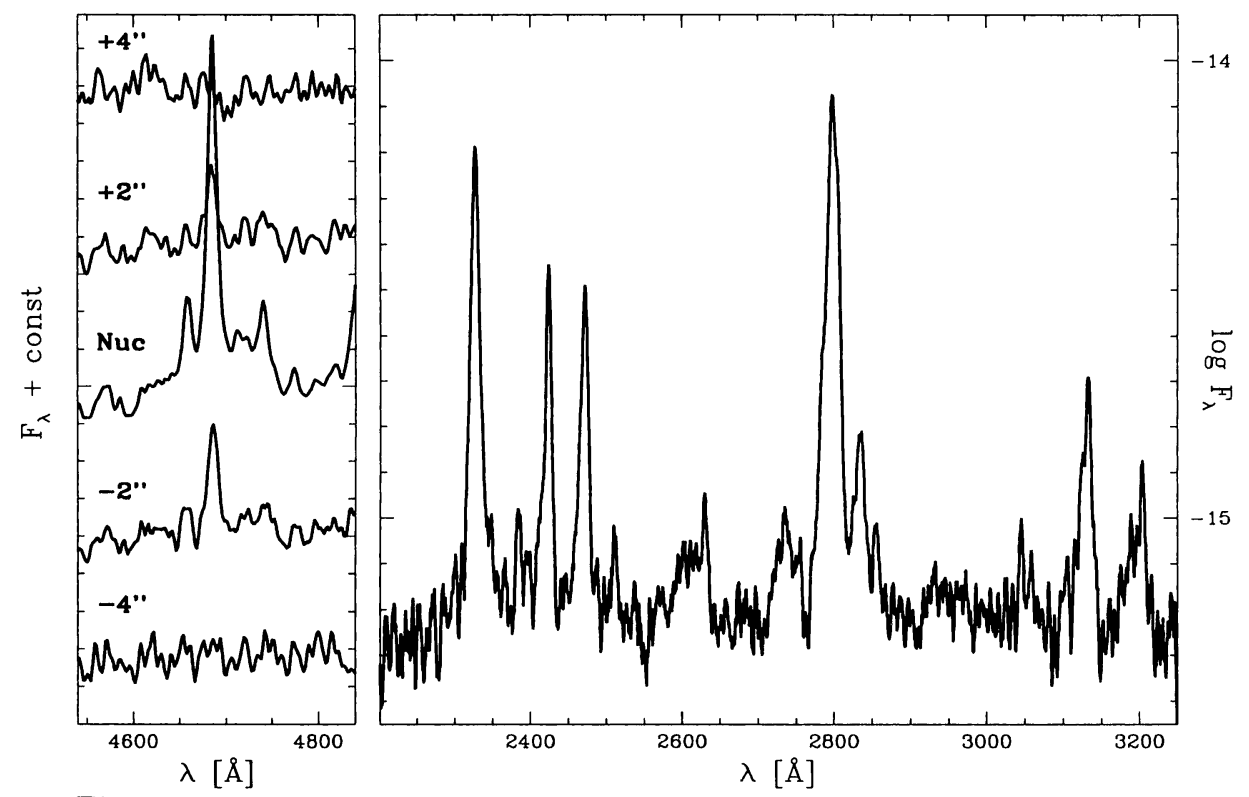

Figure 1. Left: Long-slit optical spectrum of Mrk 1210 zoomed in the region of the WR bump. Right: Near-UV HST spectrum. Most of the lines are from the the Narrow Line Region (powered by the AGN), but some (e.g., He II $\lambda 2734$, He I $\lambda 3188$, He II $\lambda 3203$ ) can be due to WR stars.

$\lambda 1240$, He II $\lambda 1640$ ) are needed to disentagle the starburst and AGN components and estimate their contributions to the overall energetics of Mrk 1210.

The detection of WR stars in AGN poses interesting and challenging questions regarding the connection between star-formation and nuclear activity. As these $\sim 3-6$ Myr starbursts are bound to evolve, its is natural to ask: what will Mrk 1210 and Mrk 477 look like 10 or 100 Myr from now? Will they still be Seyfert 2s, or will they turn into Seyfert 1s, LINERs, or dormant AGNs? Which known objects correspond to this later stage? In summary, does the age of the starburst correlate in some way with the activity level? Answers to these fundamental questions would clarify exactly what role star-formation plays in the AGN-phenomenon.

\section{References}

Cid Fernandes, R., Terlevich, R. 1995, MNRAS 272, 423

Heckman, T., et al. 1997, ApJ 482, 114

Storchi-Bergmann, T., Cid Fernandes, R., Schmitt, H. 1998, ApJ 501, 94

Tran, H. 1995, ApJ 440, 597

Tran, H., et al. 1999, ApJ in press 